\title{
The correlation between solar and geomagnetic activity - Part 3: An integral response model
}

\author{
Z. L. Du \\ Key Laboratory of Solar Activity, National Astronomical Observatories, Chinese Academy of Sciences, Beijing 100012, \\ China
}

Received: 25 January 2011 - Revised: 29 May 2011 - Accepted: 1 June 2011 - Published: 15 June 2011

\begin{abstract}
An integral response model is proposed to describe the relationship between geomagnetic activity $(a \mathrm{a}$ index) and solar activity (represented by sunspot number $R_{\mathrm{z}}$ ): The $a a$ at a given time $t$ is the integral of $R_{\mathrm{z}}$ at past times $\left(t^{\prime} \leq t\right)$ multiplied by an exponential decay factor of the time differences $\left(e^{-\left(t-t^{\prime}\right) / \tau}\right)$, where $\tau$ is the decay time scale $(\sim 40$ months). The correlation coefficient of $a a$ with the reconstructed series based on this model $\left(r_{\mathrm{f}}=0.85\right)$ is much higher than that of $a a$ with $R_{\mathrm{Z}}\left(r_{0}=0.61\right)$. If this model is applied to each solar cycle, the correlation coefficient will be higher $\left(r_{\mathrm{f}}=0.95\right)$. This model can naturally explain some phenomena related to $a a$ and $R_{\mathrm{z}}$, such as (i) the significant increase in the $a a$ index (and its baseline) over the twentieth century; (ii) the longer lag times of $a a$ to $R_{\mathrm{z}}$ at solar cycle maxima than at minima; and (iii) the variations in the correlations related to solar and Hale cycles. These results demonstrate that $a a$ depends not only on the present $R_{\mathrm{Z}}$ but also on past values. The profile of $a a$ can be better predicted from $R_{\mathrm{Z}}$ by this model than by point-point correspondence.
\end{abstract}

Keywords. Solar physics, astrophysics, and astronomy (General or miscellaneous)

\section{Introduction}

Studying the relationship between solar and geomagnetic activity is useful for understanding the origin and formation of the latter. The geomagnetic activity index $a a$, calculated from the 3-hourly $\mathrm{K}$ indices measured at two near-antipodal midlatitude stations (Mayaud, 1972), has been used for an-

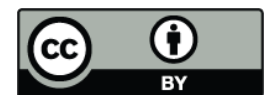

Correspondence to: Z. L. Du

(zldu@nao.cas.cn) alyzing long-term trends in the global geomagnetic activity and in its correlation with solar activity (Schatten et al., 1978; Feynman, 1982; Legrand and Simon, 1989; Nevanlinna and Kataja, 1993; Schatten and Pesnell, 1993; Russell and Mulligan, 1995; Mursula et al., 2004; Prestes et al., 2006; Cameron and Schüssler, 2007; Du et al., 2009; Lukianova et al., 2009; $\mathrm{Du}$ and Wang, 2011). It has an 11-year variation similar to that of the solar activity, as described by the Zurich relative sunspot number $\left(R_{\mathrm{z}}\right)$. In the twentieth century, there has been a significant increase in the $a a$ index (and its baseline), the reason for which, however, is unknown (Feynman and Crooker, 1978; Clilverd et al., 1998; Demetrescu and Dobrica, 2008; Lukianova et al., 2009).

The solar activity has long been recognized to be at the origin of the geomagnetic activity (Snyder et al., 1963; Russell and McPherron, 1973; Garrett et al., 1974; Feynman, 1980; Legrand and Simon, 1981). The geomagnetic activity is the result of variable current systems formed in the magnetosphere and ionosphere as a consequence of the interaction of the solar wind with the magnetosphere (Gonzalez and Tsurutani, 1987; Gonzalez et al., 1989, 1994; Demetrescu and Dobrica, 2008). The geomagnetic activity has been found to be well correlated with the solar wind speed $(v)$, the southward component $\left(B_{\mathrm{z}}\right)$ of the interplanetary magnetic field (IMF) and the product $B_{\mathrm{z}} v^{2}$ (Snyder et al., 1963; Russell and McPherron, 1973; Garrett et al., 1974; Crooker et al., 1977; Svalgaard, 1977; Feynman, 1980; Tsurutani et al., 1988; Wang and Sheeley, 2009).

Different solar and geomagnetic activities tend to peak at different times relative to the peak of each sunspot cycle. For example, the upper chromospheric activity indices (Bachmann and White, 1994) and the solar flares (Wheatland and Litvinenko, 2001; Temmer et al., 2003) tend to lag behind the sunspot number by one to several months, depending on the

Published by Copernicus Publications on behalf of the European Geosciences Union. 
index, which was interpreted in terms of active regions evolving from the photosphere upward. The solar transient activity (e.g., solar flares) dominates the rising phase (BorelloFilisetti et al., 1992), while recurrent geomagnetic activity is more frequent during the declining phase or at the minimum of solar cycle (Legrand and Simon, 1989; Sargent, 1985; Tsurutani et al., 1995; Venkatesan et al., 1991; Echer et al., 2004; Tsurutani et al., 2006; Richardson and Cane, 2002). During the last years of a cycle, the geomagnetic activity results from recurrent storms, fast solar winds, and coronal holes (Svalgaard, 1977; Legrand and Simon, 1981). The solar magnetic field that permeates the corona strongly modulates these activities (Schwenn, 2006).

A determining role in the formation and dynamics of solar activity is played by magnetic fields. Magnetic fields fill the solar atmosphere - from underlying the solar surface to the outer atmosphere, the solar corona - and have been linked with changes in total cloud cover over the Earth, which may influence global climate (Lockwood et al., 1999). Sunspots represent one of the most obvious manifestations of local magnetic fields on the Sun, the main sites of solar-activity phenomena (Moradi et al., 2010), and have been considered as a measure for the energy supply to the corona (Temmer et al., 2003). The magnetic field and its topology in the corona are closely related to the magnetic field on the solar photosphere (Sakurai, 1981; Yan and Li, 2006), and can be analyzed by potential or non-potential field extrapolation from the photospheric field (Sakurai, 1981; Tu and Marsch, 1995; Yan and $\mathrm{Li}, 2006$ ). Coronal holes are the origins of highspeed solar wind streams (Parker, 1963; Gosling and Pizzo, 1999; Tsurutani et al., 1995, 2006; Gonzalez et al., 2004). Therefore, it is natural that there are direct or indirect connections between the $a a$ geomagnetic index and the sunspot number $R_{\mathrm{z}}$.

How does the solar activity affect the geomagnetic activity, linearly or nonlinearly? In what manner? Although it has been well known that the solar activity is the main source of geomagnetic activity, there are no accurate expressions to clearly describe the relationship between them. It has been established that the magnetosphere has a significant linear response to the solar wind drivers. In the declining phase of a solar cycle, however, the dynamics of the magnetosphere exhibit a nonlinear behavior (Johnson and Wing, 2005). The increasing occurrence of high-speed solar wind streams during the declining phase of the cycle, whose reason needs to be explained, has been used to explain the decreasing trend in the correlation between $a a$ and $R_{\mathrm{Z}}$ over time (Bame et al., 1976; Borello-Filisetti et al., 1992; Mussino et al., 1994; Tsurutani et al., 1995; Kishcha et al., 1999).

What is the reason for the significant increase in the $a a$ index (and its baseline) over the twentieth century? Why does the $a a$ index tends to lag behind $R_{\mathrm{Z}}$ about $2-3$ years around a solar cycle maximum (Wang et al., 2000; Echer et al., 2004), while around a cycle minimum the lag time is small, at about 1 year (Wilson, 1990; Wang and Sheeley, 2009)? Why is the $a a$ index strongly correlated with $R_{\mathrm{Z}}$ at the rising phase of a solar cycle, while the correlation at the declining phase is weak and decreases with time (Borello-Filisetti et al., 1992; Mussino et al., 1994; Kishcha et al., 1999; Echer et al., 2004; $\mathrm{Du}, 2011 \mathrm{~b})$ ?

Conventionally, the relationship between $a a$ and $R_{\mathrm{z}}$ has been analyzed by point-point correspondence. However, some of the above questions are hardly understood and the correlation between them is unsatisfactory whether considering the time delay of $a a$ to $R_{\mathrm{z}}$ or not. This paper investigates the systematic relationship between $a a$ and $R_{\mathrm{z}}$ by an integral response model of $a a$ to $R_{\mathrm{Z}}$. In this model, the output $(a a)$ depends not only on the present input $\left(R_{\mathrm{z}}\right)$ but also on past values. Using this model, the above questions (Du, 2011b) can be naturally explained. Section 2 shows the results for all data since the onset of Cycle 12 followed by explanations for the above questions in Sect. 3. Section 4 shows the results when this model is applied to each cycle of 12 through 23. Our conclusions are discussed and summarized in Sect. 5.

\section{Results}

The data used in this study are the time series of monthly mean $a a$ geomagnetic index, representing the geomagnetic activity (Mayaud, 1972), of the reliable values since $1868^{1}$ together with the monthly mean sunspot number $\left(R_{\mathrm{z}}\right)^{2}$, representing the solar activity. To filter out high frequency variations, the data have been smoothed with a 24-month Gaussian filter. The relative weights are given by

$W(\Delta t)=\exp \left[-2 \Delta t^{2} / b^{2}\right]-\exp [-2]\left(3-2 \Delta t^{2} / b^{2}\right)$,

where $\Delta t$ is the number of months from the center and $b$ (=24 months) is the full width at half maximum (Hathaway et al., 2002). For comparison with the results of individual cycles, we employ the data from the onset of Cycle 12 (August 1878) to the end of Cycle 23 (August 2008), as shown in Fig. 1: $a a-5$ (solid line, shifted downward by 5 for clarity) and $R_{\mathrm{Z}}$ (dashed) to the right of the vertical dash-dotted line. The correlation coefficient between $a a$ and $R_{\mathrm{Z}}$ is $r_{0}=0.61$ about only one-third $\left(r_{0}^{2}=37.2 \%\right)$ of the variation in $a a$ can be explained by a linear correlation.

\subsection{The integral response model of $a a$ to $R_{z}$}

Generally, the output $O(t)$ of a system will depend not only on the present input $I(t)$, but also on past values. Approximately, $O(t)$ is a weighted sum of the previous values of $I\left(t^{\prime}\right)$, with the weights given by a response function $h\left(t-t^{\prime}\right)$,

$O(t)=\int_{-\infty}^{t} I\left(t^{\prime}\right) h\left(t-t^{\prime}\right) d t^{\prime}$.

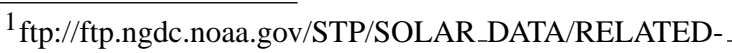
INDICES/AA_INDEX/

${ }^{2}$ http://www.ngdc.noaa.gov/stp/SOLAR/ftpsunspot-number. $\mathrm{html}$
} 


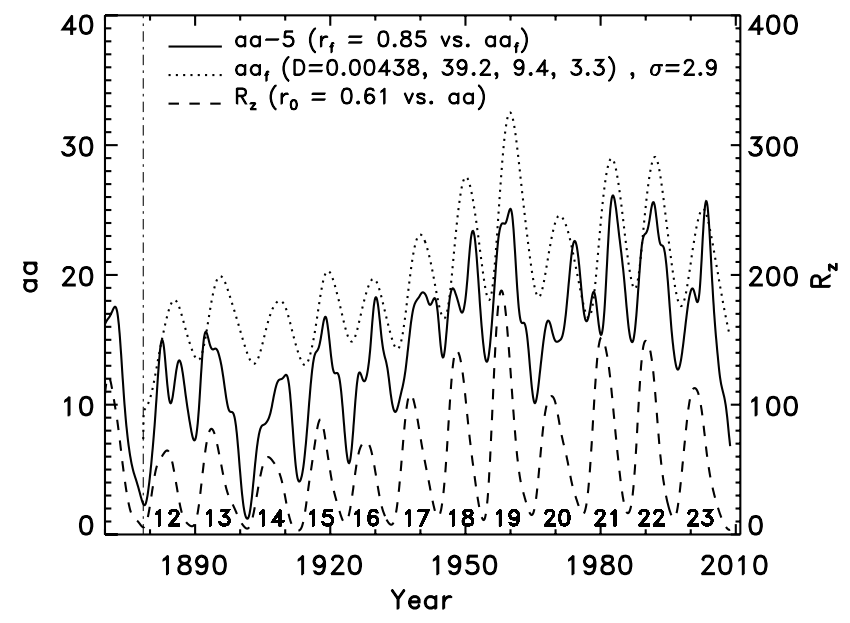

Fig. 1. Monthly mean $a a-5$ (solid) and $R_{\mathrm{Z}}$ (dashed) smoothed with a 24-month Gaussian filter. The dotted line shows the reconstructed $a a$ series $\left(a a_{\mathrm{f}}\right)$ from August 1878 through August 2008 (Cycles 12-23) by Model (7). The correlation coefficients of $a a$ with $R_{\mathrm{Z}}$ and $a a_{\mathrm{f}}$ are $r_{0}=0.61$ and $r_{\mathrm{f}}=0.85$, respectively.

In this study, the output is $a a$ and the input is $R_{\mathrm{z}}$. Now, we determine the explicit expression of Eq. (2). Suppose that at time $t=t^{\prime}$ there is an input from solar activity, which is approximately represented by $R_{\mathrm{Z}}\left(t^{\prime}\right)$, and that this input generates an output of geomagnetic activity linearly correlated with $R_{\mathrm{Z}}\left(t^{\prime}\right)$,

$a a^{\prime}\left(t^{\prime}\right)=D\left(R_{\mathrm{Z}}\left(t^{\prime}\right)+R_{0}\right)$,

where $D$ and $R_{0}$ are constants, the prime on $a a$ represents the part of $a a$ that is generated by $R_{\mathrm{Z}}\left(t^{\prime}\right)$.

This activity then undergoes a decay process according to time $t$ (due to variable current systems etc),

$$
-\Delta a a^{\prime} \propto a a^{\prime} \Delta t, \quad \text { or } \quad \frac{\partial a a^{\prime}}{\partial t}=-\frac{1}{\tau} a a^{\prime},
$$

where $\tau$ indicates the decay time scale. Its solution is

$a a^{\prime}(t)=e^{-\left(t-t^{\prime}\right) / \tau} a a^{\prime}\left(t^{\prime}\right)$,

where $a a^{\prime}\left(t^{\prime}\right)$ is the integral constant at $t=t^{\prime}$, which has been already assumed to be a linear function of $R_{\mathrm{Z}}\left(t^{\prime}\right)$ at the initial time $t=t^{\prime}$ (Eq. 3). So that,

$a a^{\prime}(t)=D\left(R_{\mathrm{Z}}\left(t^{\prime}\right)+R_{0}\right) e^{-\left(t-t^{\prime}\right) / \tau}$.

This equation means that a solar activity $\left(R_{\mathrm{Z}}\left(t^{\prime}\right)\right)$ at time $t^{\prime}$ will generate a series of geomagnetic activities $\left(a a^{\prime}(t)\right)$ in the subsequent times $\left(t>t^{\prime}\right)$ according to an exponential decay factor $\left(e^{-\left(t-t^{\prime}\right) / \tau}\right)$. In other words, the geomagnetic activity at time $t$ is generated by all the solar activities before time $t$, i.e., the summation of Eq. (6),

$$
\begin{aligned}
a a(t) & =\sum a a^{\prime}(t)+a a_{0} \\
& =D \int_{t^{\prime}=-\infty}^{t}\left[R_{\mathrm{Z}}\left(t^{\prime}\right)+R_{0}\right] e^{-\left(t-t^{\prime}\right) / \tau} d t^{\prime}+a a_{0} \\
& =D \sum_{t^{\prime}=t_{0}}^{t}\left[R_{\mathrm{Z}}\left(t^{\prime}\right)+R_{0}\right] e^{-\left(t-t^{\prime}\right) / \tau}+a a_{0} .
\end{aligned}
$$

In this expression, the input is the solar activity $\left(I\left(t^{\prime}\right) \propto\right.$ $\left.R_{\mathrm{Z}}\left(t^{\prime}\right)\right)$, the output is the geomagnetic activity $(O(t) \propto$ $a a(t))$, and the response function $h\left(t-t^{\prime}\right) \propto e^{-\left(t-t^{\prime}\right) / \tau}$. The summation is taken over from the starting time $\left(t_{0}\right)$ of the series to time $t$.

The meanings of the parameters are as follows: (i) $D$ is called "Dynamic response factor", representing the initial generation efficiency of geomagnetic activity $\partial a a_{\mathrm{i}} / \partial R_{\mathrm{z}}$; (ii) $\tau$ is called "response time scale" of $a a$ to $R_{\mathrm{Z}}$, representing that a solar activity may generate a series of geomagnetic activities in the subsequent time period of about $\tau$ (months); (iii) $a a_{0}$ is a constant, representing the geomagnetic activity generated by earlier solar activities (see Sects. 3.1 and 4.2), and (iv) $R_{0}$ represents that some weak solar activities (magnetic fields), which should be but have not been seen in the form of sunspots (the sunspots or dark pores are too small to be seen), may also generate geomagnetic activities. Penn and Livingston (2006) pointed out that the magnetic field has a threshold of 1500 Gauss, below which no dark pores formed, which represents a real physical limit for the formation of a dark spot (either a pore or a sunspot) on the solar photosphere. Weak magnetic fields may be insufficient to form sunspots, but can generate geomagnetic activities. The value of $R_{0}$ just reflects the effect of these weak fields.

\subsection{Four-parameter model}

Firstly, we use model (7) to fit the $a a$ series from the onset of Cycle 12 through the end of Cycle 23. The four parameters by a least-squares-fit are

$$
\left\{\begin{array}{l}
D=0.00438 \pm 0.00004, \\
\tau=39.2 \pm 0.4 \text { (month), } \\
a a_{0}=9.4 \pm 0.2, \\
R_{0}=3.3 \pm 1.6,
\end{array}\right.
$$

where \pm represents the standard deviation.

Using these parameters and Model (7), the $a a$ series can be reconstructed, as shown in Fig. $1\left(a a_{\mathrm{f}}\right.$, dotted line). It is seen that $a a_{\mathrm{f}}$ well reflects the profile of $a a$, the time delay of $a a$ to $R_{\mathrm{Z}}$, and the increase in $a a$ or the baseline (the $a a$ minimum of geomagnetic cycle, or simply the $a a$ minimum, $a a_{\min }$ ) over the twentieth century. The standard deviation of the reconstruction is $\sigma=2.9$. The correlation coefficient between $a a$ and the reconstructed series $\left(a a_{\mathrm{f}}\right)$ is $r_{\mathrm{f}}=0.85$, much higher than the original value $\left(r_{0}=0.61\right)$ between $a a$ and $R_{\mathrm{z}}$. This means that about two-thirds $\left(r_{f}^{2}=72.3 \%\right)$ of the variation in $a a$ can be explained by Model (7), much higher than that $\left(r_{0}^{2}=37.2 \%\right)$ for a linear correlation.

One may argue that, if considering the time delay of $a a$ to $R_{\mathrm{z}}$, the correlation between $a a$ and $R_{\mathrm{z}}$ will also be improved. To show this, we calculate the (linear) correlation function $(r)$ between $R_{\mathrm{Z}}$ and $a a$ of the lag $L=-200,-199$, ..., 200, as shown in Fig. 2a. It is seen that $r$ varies with a periodicity of about 129 months, which represents the $(\sim 11$ year) Schwabe cycle in both $R_{\mathrm{z}}$ and $a a$. The maximum value 

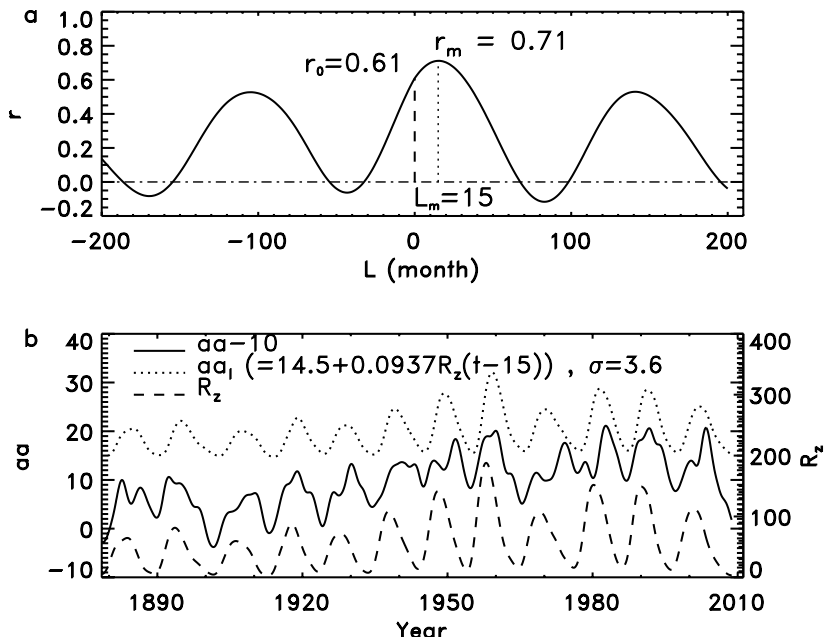

Fig. 2. (a) Correlation function between $R_{\mathrm{Z}}$ and $a a$ of the lag $L=$ $-200,-199, \ldots, 200$ (months). (b) $a a-10$ (solid), $R_{\mathrm{Z}}$ (dashed), and the reconstructed series $a a_{1}$ (dotted) from a linear fit of $a a$ to the $R_{\mathrm{Z}}$ fifteen months earlier (9).

is $r_{\mathrm{m}}=0.71$ at a lag of $L_{\mathrm{m}}=15$ (months). The best-fit equation of $a a(t)$ against $R_{\mathrm{z}}(t-15)$ is given by

$a a(t)=14.5 \pm 0.2+(0.0937 \pm 0.0022) R_{\mathrm{Z}}(t-15)$.

The standard deviation of the regression equation is $\sigma=3.6$. Figure $2 \mathrm{~b}$ shows $a a-10$ (solid, shifted downward by 10 for clarity), $R_{\mathrm{Z}}$ (dashed), and the reconstructed series $a a_{1}$ (dotted) from this linear relationship (Eq. 9). Even if considering the lag time $\left(L_{\mathrm{m}}\right)$ of $a a$ to $R_{\mathrm{Z}}$, the correlation coefficient (0.71) between $a a$ and $a a_{1}$ is still lower than that $\left(r_{\mathrm{f}}=0.85\right)$ from Model (7) in Fig. 1. In addition, although $a a_{1}$ can approximately indicate the $a a$ maxima $\left(a a_{\max }\right)$, while the reconstructed $a a$ minima from $a a_{1}$ almost keep a constant level (from 15.0 to 14.8 in the range of $[14.8,16.0]$ with a rising factor of $14.8 / 15.0 \sim 1.0)$ due to the small values of the solar minima $\left(R_{\min }\right)$ and thus can not reflect the increasing trend in the baseline $\left(a a_{\mathrm{min}}\right)$ of $a a$. Therefore, to describe the relationship between $a a$ and $R_{\mathrm{Z}}$, model (7) is more appropriate than a simple linear function.

\subsection{Three-parameter model}

When using Model (7) to fit the $a a$ time series in the above section, the $R_{0}$ value is small (3.3). Therefore, we neglect $R_{0}$ and use the following three-parameter expression to refit the $a a$ series for Cycles 12-23,

$$
\begin{aligned}
a a(t) & =D \int_{t^{\prime}=-\infty}^{t} R_{\mathrm{Z}}\left(t^{\prime}\right) e^{-\left(t-t^{\prime}\right) / \tau} d t^{\prime}+a a_{0} \\
& =D \sum_{t^{\prime}=t_{0}}^{t} R_{\mathrm{Z}}\left(t^{\prime}\right) e^{-\left(t-t^{\prime}\right) / \tau}+a a_{0} .
\end{aligned}
$$

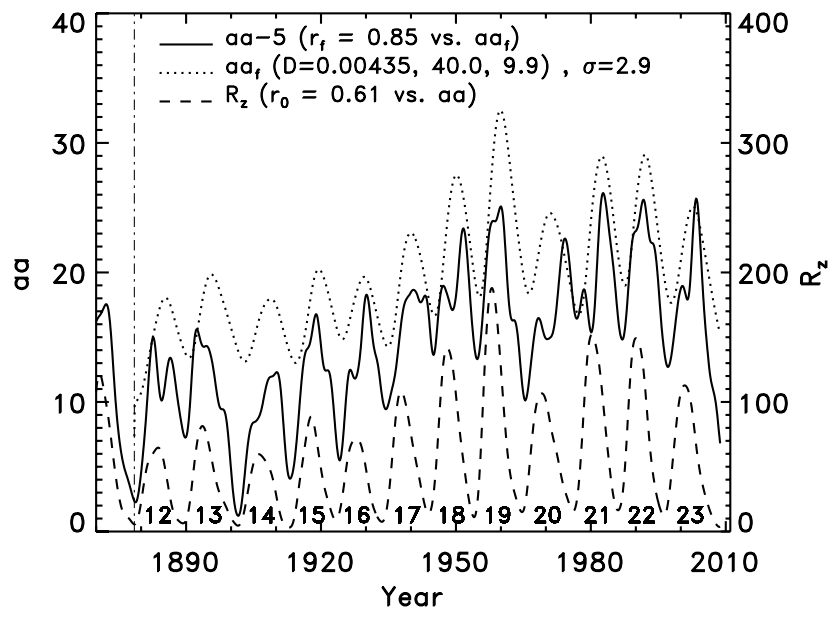

Fig. 3. Similar to Fig. 1 but using three-parameter Model (10). The correlation coefficient of $a a$ with the reconstructed series $a a_{\mathrm{f}}$ is also $r_{\mathrm{f}}=0.85$.

The reconstructed $a a$ series $\left(a a_{\mathrm{f}}\right.$, dotted) is shown in Fig. 3 . The three parameters based on this model,

$\left\{\begin{array}{l}D=0.00435 \pm 0.00004, \\ \tau=40.0 \pm 0.4 \text { (month), } \\ a a_{0}=9.9 \pm 0.1,\end{array}\right.$

have no significant changes compared with those for the fourparameter Model (8). The standard deviation of the reconstruction keeps the same level, $\sigma=2.9$. As the $R_{0}$ value in Eq. (8) is very small (3.3) and the correlation coefficient between $a a$ and the reconstructed series $a a_{\mathrm{f}}$ in this case $\left(r_{\mathrm{f}}=0.85\right)$ is equal to that in the case for four-parameter model in Fig. 1, the effect of three-parameter Model (10) is equivalent to that of four-parameter Model (7). Therefore, we will use the three-parameter Model (10) in the following sections.

\section{Explanations for some correlations of $a a$ with $R_{\mathrm{z}}$}

It is seen in Fig. 3 that the reconstructed series $\left(a a_{\mathrm{f}}\right)$ generally reflects the profile of $a a$, the time delay of $a a$ to $R_{\mathrm{z}}$, and the increase in $a a$ over the twentieth century. Therefore, Model (10) well represents the response of geomagnetic to solar activity, and can naturally explain in part the relationship between $a a$ and $R_{z}$.

For convenience, Table 1 lists the maximum amplitude of sunspot cycle $\left(R_{\max }\right)$, the preceding $a a$ minimum $\left(a a_{\min }\right)$, the $a a$ maximum of geomagnetic cycle $\left(a a_{\max }\right)$, the lag times of $a a$ to $R_{\mathrm{Z}}$ at solar minimum $\left(L_{\mathrm{min}}\right)$ and at solar maximum $\left(L_{\max }\right)$, and the corresponding values $\left(a a_{\min , \mathrm{f}}, a a_{\max , \mathrm{f}}\right.$, $\left.L_{\text {min,f }}, L_{\max , \mathrm{f}}\right)$ from the reconstructed series $a a_{\mathrm{f}}$ in Fig. 3 . The last two rows in Table 1 indicate the averages of these values and the correlation coefficients of these values with $R_{\max }$. 
Table 1. Geomagnetic minimum $\left(a a_{\min }\right)$, maximum $a a\left(a a_{\max }\right)$, lag times of $a a$ to $R_{\mathrm{Z}}$ at minimum $\left(L_{\min }\right)$ and at maximum $\left(L_{\max }\right)$ for Cycles $n=12-23$, and the corresponding values $\left(a a_{\min , \mathrm{f}}, a a_{\max , \mathrm{f}}, L_{\min , \mathrm{f}}, L_{\max , \mathrm{f}}\right)$ from the reconstructed series (10). The last two rows show the averages of these values and the correlation coefficients $(r)$ of these values with the maximum amplitude $\left(R_{\text {max }}\right)$ of sunspot cycle.

\begin{tabular}{|c|c|c|c|c|c|c|c|c|c|}
\hline \multirow[b]{2}{*}{$n$} & \multirow[b]{2}{*}{$R_{\max }$} & \multicolumn{4}{|c|}{ Observed } & \multicolumn{4}{|c|}{ Fitted } \\
\hline & & $a a_{\min }$ & $a a_{\max }$ & $L_{\min }$ & $L_{\max }$ & $a a_{\min , \mathrm{f}}$ & $a a_{\max , \mathrm{f}}$ & $L_{\min , \mathrm{f}}$ & $L_{\max , \mathrm{f}}$ \\
\hline 12 & 64.7 & 7.3 & 20.0 & 3 & -14 & 7.3 & 18.0 & 0 & 19 \\
\hline 13 & 81.4 & 12.3 & 20.6 & 6 & -15 & 13.4 & 19.8 & 16 & 23 \\
\hline 14 & 59.6 & 6.2 & 17.3 & 0 & 47 & 13.1 & 18.0 & 18 & 31 \\
\hline 15 & 88.6 & 9.1 & 21.8 & 3 & 13 & 13.0 & 20.2 & 19 & 20 \\
\hline 16 & 71.6 & 10.5 & 23.3 & 11 & 27 & 14.8 & 19.7 & 18 & 21 \\
\hline 17 & 108.2 & 14.4 & 23.7 & 11 & 33 & 14.3 & 23.1 & 17 & 26 \\
\hline 18 & 141.7 & 18.6 & 28.4 & 13 & 43 & 16.7 & 27.5 & 16 & 24 \\
\hline 19 & 188.0 & 18.3 & 30.1 & 9 & 24 & 18.1 & 32.5 & 15 & 23 \\
\hline 20 & 106.6 & 15.1 & 27.6 & 9 & 62 & 18.4 & 24.6 & 19 & 22 \\
\hline 21 & 151.8 & 20.4 & 31.1 & 48 & 30 & 16.7 & 29.0 & 15 & 26 \\
\hline 22 & 149.2 & 19.8 & 30.6 & 12 & 20 & 19.0 & 29.1 & 19 & 25 \\
\hline 23 & 112.8 & 17.7 & 30.7 & 11 & 31 & 17.7 & 25.1 & 19 & 23 \\
\hline $\bar{x}$ & 110.4 & 14.1 & 25.4 & 11 & 25 & 15.2 & 23.9 & 16 & 24 \\
\hline$r$ & 1.00 & 0.89 & 0.86 & 0.50 & 0.21 & 0.73 & 0.99 & 0.23 & 0.08 \\
\hline
\end{tabular}

\subsection{The increase in $a a$ over the twentieth century}

It is well known that, in the twentieth century, there has been a significant increase in the $a a$ index (and its baseline), the reason for which, however, is unknown (Feynman and Crooker, 1978; Clilverd et al., 1998; Demetrescu and Dobrica, 2008; Lukianova et al., 2009).

Figure $4 \mathrm{a}$ shows the values of $a a_{\min }$ (solid), $a a_{\min , \mathrm{f}}$ (dotted) and $R_{\max }$ (dashed). It is clearly seen that $a a_{\min , \mathrm{f}}$ is well correlated with $a a_{\min }(r=0.82)$ and well reflects the increasing trend in $a a_{\min }$ (with rising factors of 17.7/7.4 vs. 17.7/7.3 $\sim 2.4)$. Both values $\left(a a_{\min }, a a_{\min , \mathrm{f}}\right)$ are well correlated with $R_{\text {max }}(r=0.89,0.73)$.

Figure $4 \mathrm{~b}$ shows the values of $a a_{\max }$ (solid), $a a_{\max , f}$ (dotted) and $R_{\max }$ (dashed). It can also be seen that $a a_{\max , \mathrm{f}}$ is highly correlated with $a a_{\max }(r=0.91)$ and well reflects the increasing trend in $a a_{\max }$ (with rising factors of 25.1/18.0 1.4 vs. $30.7 / 20.0 \sim 1.5)$. The correlation coefficients of these two values $\left(a a_{\max }, a a_{\max , \mathrm{f}}\right)$ with $R_{\max }$ are also very high $(r=0.86,0.99)$.

In Model (10), the $a a$ index is viewed as the total contributions from all the solar activities $\left(R_{\mathrm{z}}\right)$ at the present and in the past times. Therefore, the increasing trends in $a a, a a_{\max }$ and $a a_{\min }$ (baseline) are caused by the increasing trend in $R_{\mathrm{Z}}$ (Fig. 3). This confirms the suggestions that the change in $a a$ is caused by an increase in solar magnetic activity over the last century (Lockwood et al., 1999), and that the increasing trend in magnetic storm is most likely caused by solar activity (Clilverd et al., 1998). As the value of $a a_{\min }$ and its increasing trend can be well reconstructed by Model (10) to-
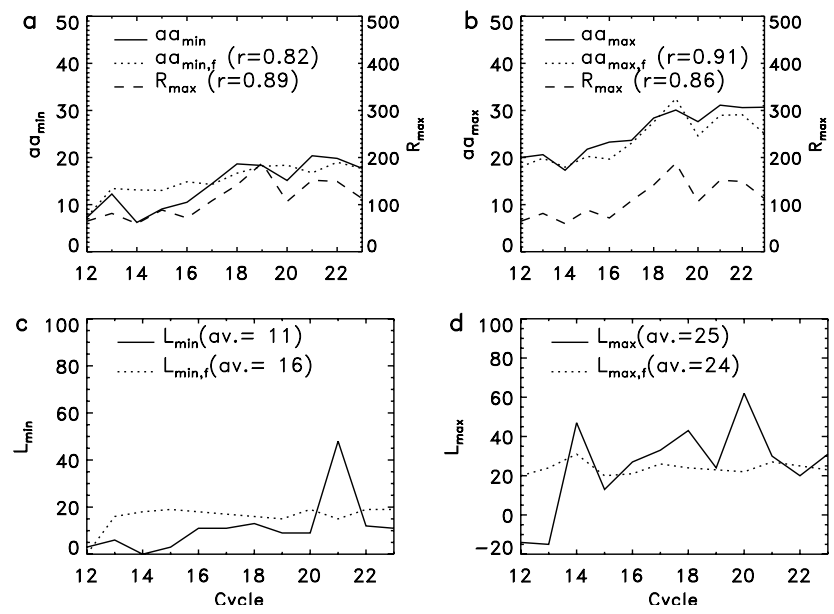

Fig. 4. The observed (a) geomagnetic minimum $\left(a a_{\min }\right)$, (b) maximum $a a\left(a a_{\max }\right)$, (c) lag times of $a a$ to $R_{\mathrm{Z}}$ at solar minimum $\left(L_{\min }\right)$ and (d) at maximum $\left(L_{\max }\right)$ for Cycles $n=12-23$ are shown by solid lines, and the corresponding values $\left(a a_{\min , \mathrm{f}}, a a_{\max , \mathrm{f}}, L_{\min , \mathrm{f}}\right.$,

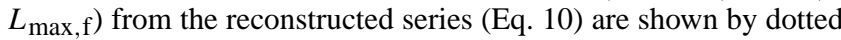
lines. The dashed lines in (a) and (b) show the maximum amplitude $\left(R_{\max }\right)$ of sunspot cycle for comparison. The correlation coefficients of $a a_{\min }$ with $a a_{\min , \mathrm{f}}$ and $R_{\max }$ are $r=0.82$ and 0.89 , respectively. The correlation coefficients of $a a_{\max }$ with $a a_{\max , \mathrm{f}}$ and $R_{\max }$ are $r=0.91$ and 0.86 , respectively.

gether with the increasing trend in $R_{\mathrm{z}}, a a_{\min }$ represents the geomagnetic activity generated by earlier solar activities. 


\subsection{The longer lag times of $a a$ to $R_{\mathrm{Z}}$ at solar maxima than at minima}

The $a a$ index tends to lag behind $R_{\mathrm{z}}$ about 2-3 years around a solar maximum (Wang et al., 2000; Echer et al., 2004), and about 1 year around a solar minimum (Legrand and Simon, 1981; Wilson, 1990; Wang and Sheeley, 2009).

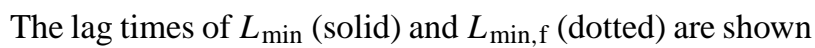
in Fig. 4c, and those of $L_{\max }$ (solid) and $L_{\max , \mathrm{f}}$ (dotted) are shown in Fig. 4d. It is seen that the average lag time at solar minima from the reconstructed series $\left(\bar{L}_{\mathrm{min}, \mathrm{f}}=16\right)$ is near to the observed one $\left(\bar{L}_{\min }=11\right)$, and that the average lag time at solar maxima from the reconstructed series $\left(\bar{L}_{\mathrm{max}, \mathrm{f}}=24\right)$ is close to the observed one $\left(\bar{L}_{\max }=25\right)$. In addition, the reconstructed series can also indicate the smaller average lag time at solar minima than at solar maxima $(16<24$ vs. $11<25)$. The weak correlations between the fitted values $\left(L_{\min , \mathrm{f}}\right.$ or $\left.L_{\max , \mathrm{f}}\right)$ and the observed values $\left(L_{\min }\right.$ or $\left.L_{\max }\right)$ reflect the fact that $a a$ has several solar sources (solar flares, coronal mass ejections and solar winds etc.), and that these sources peak at different times relative to $R_{\mathrm{z}}$ for each cycle and generate the geomagnetic activities with different lag times (see Discussions).

At a solar maximum, a part of $a a$ is contributed from the solar activities $\left(R_{\mathrm{Z}}\right)$ during the rising phase of the current cycle, which are weaker than the maximum value $\left(R_{\max }\right)$. These smaller values of $R_{\mathrm{Z}}$ lead the $a a$ index not to reach its maximum at the same time of $R_{\max }$. After the timing of $R_{\max }$, a part of $a a$ is contributed from the values of $R_{\mathrm{Z}}$ around the maximum $\left(R_{\max }\right)$, which make the $a a$ index larger than that it should have been. So the $a a$ index reaches its maximum at a time later than the timing of $R_{\max }$.

At a solar minimum, a part of $a a$ is contributed from the solar activities $\left(R_{\mathrm{Z}}\right)$ during the declining phase of the preceding cycle, which are stronger than the minimum value $\left(R_{\min }\right)$. These larger values of $R_{\mathrm{Z}}$ lead the $a a$ index not to reach its minimum at the same time of $R_{\min }$. After the timing of $R_{\min }$, a part of $a a$ is contributed from the values of $R_{\mathrm{Z}}$ around the minimum $\left(R_{\min }\right)$, which make the $a a$ index smaller than that it should have been. So the $a a$ index reaches its minimum at a time later than the timing of $R_{\min }$.

Because the stronger the previous $R_{\mathrm{Z}}$ values, the more they contribute to the subsequent $a a$ values, and the longer the lag time of $a a$ to $R_{\mathrm{Z}}(10)$. The values of $R_{\mathrm{Z}}$ around a solar maximum are much larger than those around the preceding minimum (and $R_{\mathrm{Z}}$ changes more slowly during the declining phase near the minimum than near the maximum). Therefore, the lag times of $a a$ to $R_{\mathrm{Z}}$ near solar maxima (about two years) are longer than those near solar minima (about one year). While the linear relationship between $a a$ and $R_{\mathrm{Z}}$ (Fig. 2) can not indicate the longer lag times of $a a$ to $R_{\mathrm{Z}}$ at solar maxima and the shorter lag times at solar minima. The sharp increase in lag time in cycle 14 (Fig. 4d) is mainly due to the higher maxima in Cycles 12-13 than that in Cycle 14.

\subsection{The stronger correlations between $a a$ and $R_{\mathrm{Z}}$ at ris- ing phases than at declining phases}

According to Model (10), $a a(t)$ at time $t$ comes from the total contributions of $R_{\mathrm{Z}}(t-\Delta t)$ at various $\Delta t$ with a decay factor of $e^{-\Delta t / \tau}$. The longer the time interval of $R_{\mathrm{Z}}$ preceding $a a$, the less its contribution.

During the rising phase of a solar cycle, the $a a$ value is contributed from two parts of $R_{\mathrm{Z}}$ : one is that during the same rising phase, and another is that during the previous declining phase (for simplicity, we neglect the less contributions from the even earlier data). The former varies approximately linearly with time in an ascending way and has a linear response to $a a$ that makes the correlation of $a a$ with $R_{\mathrm{Z}}$ positive. The latter has a longer time delay $\Delta t$ and so contributes less to $a a$ than the former. Thus, the correlation between $a a$ and $R_{\mathrm{Z}}$ at the rising phase of a solar cycle is strong (Du, 2011b).

During the declining phase of a solar cycle, there are also two parts of $R_{\mathrm{Z}}$ that contribute to $a a$ : one is that during the same declining phase, and another is that during the preceding rising phase. The former varies approximately linearly with time in a descending way and has a roughly linear response to $a a$ that makes the correlation of $a a$ with $R_{\mathrm{z}}$ positive. However, the latter varies with time in an opposite ascending way which contributes a negative correlation. Because the values of $R_{\mathrm{Z}}$ around a solar maximum are much larger than those around a solar minimum, the contribution of the negative correlation around the solar maximum is larger than that around the solar minimum. Thus, the correlation between $a a$ and $R_{\mathrm{Z}}$ at the declining phase of a solar cycle is weak (Du, 2011b).

\subsection{The decreasing trend in the correlation between $a a$ and $R_{\mathbf{z}}$}

The decreasing trend in the correlation between $a a$ and $R_{\mathrm{Z}}$ over time is due to the increasing trend in $R_{\mathrm{Z}}$ (solar magnetic activity) over the last century (Fig. 3). The values of $R_{\mathrm{Z}}$ around solar minima have no significant changes from $\mathrm{Cy}-$ cles 12 to 23, so there are no significant variations in the correlations at rising phases according to Eq. (10) and Sect. 3.3. However, the increasing trend in the values of $R_{\mathrm{Z}}$ around solar maxima ( $R_{\max }$ in Fig. $\left.4 \mathrm{~b}\right)$ contributes increasing negative correlations of $a a$ with $R_{\mathrm{Z}}$ at the following declining phases. This leads to a descending trend in the correlations at declining phases, which finally makes the correlation of $a a$ with $R_{\mathrm{Z}}$ decease for each cycle or for a given time window ( $\mathrm{Du}$, 2011b).

\subsection{The increasing trend in the lag time of $a a$ to $R_{\mathrm{Z}}$}

The increasing trend in the lag time of $a a$ to $R_{\mathrm{Z}}$ is also due to the increasing trend in $R_{\mathrm{Z}}$ over the last century. The larger the values of $R_{\mathrm{Z}}$ around solar maxima, the more their effects on the $a a$ values during the declining phases, and the longer the 


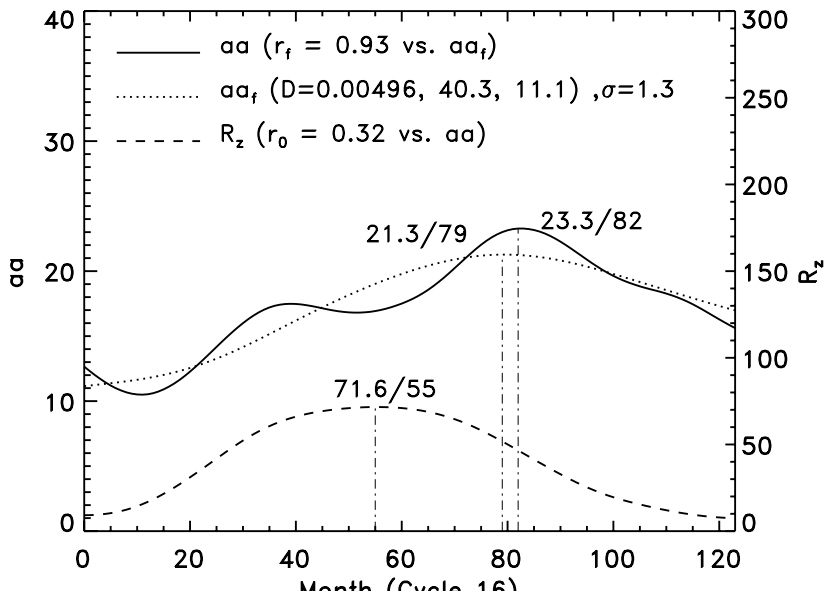

Fig. 5. Results for Cycle 16 (from April 1923 to July 1933), aa (solid), $R_{\mathrm{Z}}$ (dashed), and the reconstructed series $a a_{\mathrm{f}}$ (dotted) from Model (10). The correlation coefficients of $a a$ with $R_{\mathrm{Z}}$ and $a a_{\mathrm{f}}$ are $r_{0}=0.32$ and $r_{\mathrm{f}}=0.93$, respectively. The peak sizes/time intervals (in months) of these peaks from the minimum are also labelled.

lag time of $a a$ to $R_{\mathrm{z}}$. So, the increasing trend in $R_{\mathrm{z}}$ around solar maxima produces an increasing trend in the lag time of $a a$ to $R_{\mathrm{Z}}$ during the declining phases (besides the decreasing correlation of aa with $R_{\mathrm{Z}}$ ).

\subsection{The turning point of the correlation of aa with $\boldsymbol{R}_{\mathrm{z}}$ around Cycle 19}

The $R_{\mathrm{Z}}$ values show a roughly increasing trend from Cycles 12 to 19 and a roughly declining trend since Cycle 19 (Fig. 3). This is the reasons for the decreasing trend in the correlation between $a a$ and $R_{\mathrm{Z}}$ and the increasing lag time of $a a$ to $R_{\mathrm{z}}$ before Cycle 19 (Du, 2011b). These trends changed since Cycle 19 (around 1958).

\section{The results for modelling each cycle of 12-23}

In this section, Model (10) is applied to each Cycle of 1223. Considering that the values of $R_{\mathrm{z}}$ around a solar minimum are small and generate less geomagnetic activities than during other times, and that the activities before the solar minimum will undergo longer decay times, we take the starting time in the summation of Model (10) as the timing of the previous solar minimum $\left(t_{0}\right)$.

\subsection{For an individual Cycle 16}

Firstly, we apply Model (10) to an arbitrary solar Cycle 16 as an example. Figure 5 shows the time series of a (solid), $R_{\mathrm{z}}$ (dashed) for Cycle 16 (from April 1923 to July 1933), and the reconstructed series ( $a a_{\mathrm{f}}$, dotted).

It is seen from Fig. 5 that the reconstructed series $\left(a a_{\mathrm{f}}\right)$ well reflects the profile of $a a$ and the time delay of $a a_{\max }$
Table 2. Fitted parameters of $D, \tau$ and $a a_{0}$, and the correlation coefficients of $a a$ with $R_{\mathrm{Z}}\left(r_{0}\right)$ and $a a_{\mathrm{f}}\left(r_{\mathrm{f}}\right)$ for Cycles $n=12-23$.

\begin{tabular}{ccrrrrr}
\hline$n$ & $t_{0}$ & $10^{3} D$ & $\tau$ & $a a_{0}$ & $r_{0}$ & $r_{\mathrm{f}}$ \\
\hline 12 & Jul 1878 & 7.10 & 24.0 & 9.4 & 0.56 & 0.83 \\
13 & May 1889 & 76.26 & 1.3 & 9.9 & 0.90 & 0.90 \\
14 & Aug 1901 & 6.62 & 27.6 & 8.0 & 0.55 & 0.92 \\
15 & Nov 1912 & 10.59 & 12.8 & 10.8 & 0.84 & 0.96 \\
16 & Apr 1923 & 4.96 & 40.3 & 11.1 & 0.32 & 0.93 \\
17 & Jul 1933 & 2.67 & 58.0 & 14.6 & 0.30 & 0.99 \\
18 & Dec 1943 & 1.20 & 53.2 & 19.9 & 0.17 & 0.70 \\
19 & Jan 1954 & 6.89 & 10.1 & 17.6 & 0.88 & 0.98 \\
20 & Sep 1964 & 1.02 & 137.0 & 16.6 & -0.18 & 0.83 \\
21 & Feb 1976 & 1.35 & 58.5 & 20.6 & -0.00 & 0.71 \\
22 & Jan 1986 & 5.17 & 15.3 & 19.5 & 0.76 & 0.96 \\
23 & Apr 1996 & 6.82 & 17.2 & 15.1 & 0.65 & 0.84 \\
\hline $\bar{x}$ & & 10.88 & 37.9 & 14.4 & 0.48 & 0.88 \\
\hline
\end{tabular}

(23.3) to $R_{\max }$ (71.6): $79-55=24$ vs. $82-55=27$. The three parameters in Model (10) for Cycle 16 are $D=4.96 \times$ $10^{-3}, \tau=40.3$ (months) and $a a_{0}=11.1$. The correlation coefficient of $a a$ with $a a_{\mathrm{f}}\left(r_{\mathrm{f}}=0.93\right)$ is much higher than that ( $r_{0}=0.32$ ) of $a a$ with $R_{\mathrm{Z}}$. Besides, these results are not sensitive to the accurate definition of the timings of solar minima - the results remain nearly the same even if the starting time $\left(t_{0}\right)$ shifts a few months forward or backward.

\subsection{The fitted parameters for Cycles 12-23}

For each Cycle of $n=12-16$, we use Model (10) to fit the $a a$ index as done in the previous section. The results for all individual Cycles of $n=12-16$ are shown in Fig. 10. The three parameters are listed in Table 2 and shown in Fig. 6.

It should be noted in Fig. 6a that the parameters $D$ (solid) and $\tau$ (dotted) tend to vary in an opposite trend: $D$ increases while $\tau$ decreases, and vice versa. The correlation coefficient between them is $r=-0.41$ (or -0.87 if not considering the two outliers of Cycles 13 and 20). It represents the fact that the faster the energy transfer from solar activity to geomagnetic activity (via solar winds, for example) in a solar cycle, the less time the energy transfer needs. The average $D$ and $\tau$ are $\bar{D}=10.88 \times 10^{-3}$ and $\bar{\tau}=37.9$ (months), respectively, implying that a solar activity will affects the geomagnetic activity in the subsequent time period of about 38 months (3 years) on average.

The values of $a a_{0}$ (Fig. 6b, shifted downward by 5 for clarity) are all positive, almost all larger than the basis value (9.9) in Fig. 3, and, most importantly, vary in an increasing trend. In Fig. $6 \mathrm{~b}$ are also plotted the maximum amplitude of sunspot cycle $\left(R_{\max }\right.$, dashed) and the preceding aa minimum $\left(a a_{\mathrm{min}}\right.$, dotted). It should be noted that $a a_{0}$ is highly correlated with both $a a_{\min }(r=0.95)$ and $R_{\max }(r=0.89)$. The average $a a_{0}$ 

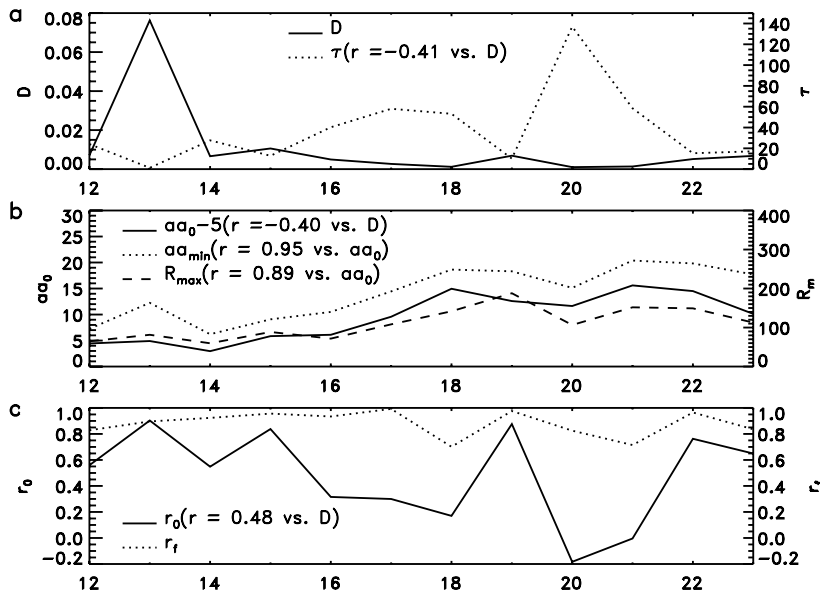

Fig. 6. Parameters for Cycles $n=12-23$ : (a) for $D$ (solid) and $\tau$ (dotted); (b) for $a a_{0}-5$ (solid), $a a_{\min }$ (dotted), and $R_{\max }$ (dashed). (c) The correlation coefficient of $a a$ with $R_{\mathrm{Z}}\left(r_{0}\right.$, solid) and that of $a a$ with $a a_{\mathrm{f}}\left(r_{\mathrm{f}}\right.$, dotted). The correlations of $D$ with $\tau, a a_{0}$, and $r_{0}$ are $r=-0.41,-0.40$, and 0.48 , respectively. The correlations of $a a_{0}$ with $a a_{\min }$ and $R_{\max }$ are $r=0.95$ and 0.89 , respectively.

$\left(\overline{a a}_{0}=14.4\right)$ is close to the average $a a_{\min }\left(\overline{a a}_{\min }=14.1\right)$. The value of $a a_{0}$ well reflects the level of and variation in $a a_{\text {min }}$. These facts imply that, besides the $R_{\mathrm{z}}$ values in the current cycle, the $R_{\mathrm{z}}$ values in the previous cycles also generate geomagnetic activities in the current cycle, and that $a a_{0}$, like $a a_{\mathrm{min}}$, is generated by the earlier $R_{\mathrm{z}}$ values.

Figure $6 \mathrm{c}$ shows the correlation coefficient of $a a$ with $R_{\mathrm{Z}}$ $\left(r_{0}\right.$, solid) and that of $a a$ with $a a_{\mathrm{f}}\left(r_{\mathrm{f}}\right.$, dotted) for each Cycle of $n=12-16$ (Table 1$)$. The average $r_{\mathrm{f}}\left(\bar{r}_{\mathrm{f}}=0.88\right)$ is much higher than the average $r_{0}\left(\bar{r}_{0}=0.48\right)$. Therefore, Model (10) reflects the relationship between solar and geomagnetic activities in a more reliable way than using a simple linear function.

The correlation coefficients of $r_{0}$ with $D$ and $\tau$ are $r=$ 0.48 (or 0.85 if not considering Cycles 13 and 20) and -0.91 , respectively. Therefore, $D$ reflects the linear correlation and $\tau$ reflects the nonlinear correlation of $a a$ with $R_{\mathrm{z}}$. Besides, $\tau$ is well correlated with $L_{\max }(0.70)$, and thus $\tau$ is related to the time delay of $a a_{\max }$ to $R_{\max }$.

\subsection{The reconstructed $a$ a series for Cycles 12-23}

Combing the results of Cycles 12-23 in the above section, the $a a$ series from Cycles 12 through 23 can be reconstructed $\left(a a_{\mathrm{f}}\right)$, as shown in Fig. 7 (dotted line).

The standard deviation of the reconstruction is $\sigma=1.8$. The correlation coefficient of $a a$ (solid, shifted downward by 5 for clarity) with $a a_{\mathrm{f}}\left(r_{\mathrm{f}}=0.95\right)$ is higher than the previous value (0.85 in Fig. 1), and much higher than that of $a a$ with $R_{\mathrm{Z}}\left(r_{0}=0.61\right)$. This means that about $r_{f}^{2}=90.3 \%$ of the variation in $a a$ can be explained by Model (10).

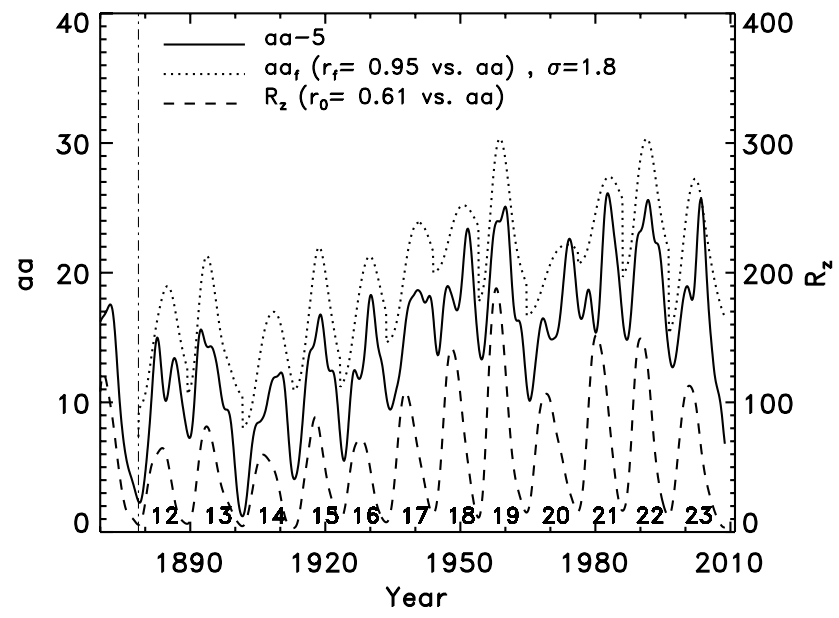

Fig. 7. Time series of $a a-5$ (solid) and $R_{\mathrm{Z}}$ (dashed) since January 1870. The dotted line shows the reconstructed $a a$ series $\left(a a_{\mathrm{f}}\right)$ from each Cycle of 12-23 by Model (10). The correlation coefficients of $a a$ with $R_{\mathrm{Z}}$ and $a a_{\mathrm{f}}$ are $r_{0}=0.61$ and $r_{\mathrm{f}}=0.95$, respectively.

\subsection{Correlations for even- and odd-numbered cycles}

An even-numbered cycle is preferentially paired with the following odd-numbered one (Wilson, 1988), constituting a Hale cycle of even-odd cycle pairs: an odd-numbered cycle tends to be stronger than the previous even-numbered one, the so-called G-O rule (Gnevyshev and Ohl, 1948; Wilson, 1988). This rule is also applicable to parameter $D$ : an oddnumbered cycle tends to have a larger $D$ than the previous even-numbered one (Table 2), with only one exception of the E-O pair of Cycles 16-17. A similar rule is also applicable to parameter $\tau$ : an odd-numbered cycle tends to have a shorter $\tau$ than the previous even-numbered one (Table 2 ), with only two exceptions of the E-O pairs of 16-17 and 22-23.

The solar maximum $\left(R_{\max }\right)$ is positively correlated with $\tau$ of the previous cycle ( $r=0.68$, or 0.82 if not considering $\mathrm{Cy}$ cle 20), and reversely correlated with $D$ of the previous cycle $(r=-0.57$, or -0.93 if not considering Cycle 13). These can explain the following phenomena.

As $D$ reflects the linear correlation and $\tau$ reflects the nonlinear correlation of $a a$ with $R_{\mathrm{z}}$, both the larger $D$ and the shorter $\tau$ imply a stronger correlation (shorter lag time) for a odd-numbered cycle than for the previous even-numbered cycle. This is consistent with the idea of the more decays for even-numbered cycles than for odd-numbered cycles ( Du, 2011a). Therefore, an odd-numbered cycle tends to have a stronger correlation of $a a$ with $R_{\mathrm{Z}}$ and a shorter lag time of $a a$ to $R_{\mathrm{Z}}$ than the previous even-numbered cycle (Du, 2011b). This cycle asymmetry has been noted by Stamper et al. (1999) that the solar wind speed peaks strongly in the declining phase of even-numbered cycles. 
Table 3. Parameters for Hale cycles $(H)$.

\begin{tabular}{ccrrr}
\hline$H$ & $n$ & $R_{\mathrm{H}}$ & $10^{3} D_{\mathrm{H}}$ & $\tau_{\mathrm{H}}$ \\
\hline 7 & $12-13$ & 73.1 & 41.68 & 12 \\
8 & $14-15$ & 74.1 & 8.61 & 21 \\
9 & $16-17$ & 89.9 & 3.82 & 49 \\
10 & $18-19$ & 164.9 & 4.01 & 32 \\
11 & $20-21$ & 129.2 & 1.19 & 98 \\
12 & $22-23$ & 131.0 & 6.00 & 16 \\
\hline
\end{tabular}

\subsection{Correlations for Hale cycles}

The above correlations can also explain the variation in the correlation of $a a$ with $R_{\mathrm{z}}$ for the Hale cycles. The even-odd cycle pairs can be numbered as the Hale cycles $(H)$ such that $H=7$ for Cycles $n=12-13, H=8$ for $n=14-15, \cdots$, $H=12$ for $n=22-23$. The averages of $R_{\mathrm{z}}, D$ and $\tau$ for $H$ cycles are listed in Table 3 such that $R_{\mathrm{H}}(7)=\left[R_{\max }(12)+\right.$ $\left.R_{\text {max }}(13)\right] / 2$, etc.

It is seen in Table 3 that an even-numbered $H$-cycle tends to be stronger than the previous odd-numbered one. Since $H=8$, an even-numbered $H$-cycle tends to have a larger $D$ and a shorter $\tau$ than the neighboring odd-numbered $H$ cycle. Therefore, an even-numbered $H$-cycle tends to have a stronger correlation and a shorter lag time of $a a$ to $R_{\mathrm{Z}}$ than the neighboring odd-numbered $H$-cycle (Du, 2011b).

\section{Discussions and conclusions}

It has been known that there are two main solar sources of geomagnetic activity (Legrand and Simon, 1981, 1989; Gonzalez and Tsurutani, 1987; Gonzalez et al., 2004; Venkatesan et al., 1991; Echer et al., 2004; Tsurutani et al., 2006). One source (coronal mass ejections or CMEs) has a frequency of occurrence that is in phase with the sunspot cycle while the second source (high-speed solar wind streams) is out of phase with the sunspot cycle. Feynman (1982), through analyzing the relationship between the annual $a a$ and $R_{\mathrm{Z}}$ from 1869 to 1975 , decomposed $a a$ into two equally strong periodic components: one (the "short lived" $\mathrm{R}$ component) associated with solar flares, prominence eruptions, and CMEs which follows the solar activity cycle and a second component (the "slowly varying" I component) associated with recurrent high speed solar wind streams which is out of phase with the solar activity cycle (Hathaway and Wilson, 2006). Legrand and Simon (1989) classified the geomagnetic activity ( $a a$ index) in four classes related to solar activity: (1) the magnetic quiet activity due to slow solar wind flowing around the magnetosphere, (2) the recurrent activity related to high wind speed solar wind, (3) the fluctuating activity related to fluctuating solar wind and (4) the shock activity due to shock events (CMEs).
A primary physical mechanism for energy transfer from the solar wind to the magnetosphere is magnetic reconnection between the interplanetary magnetic field (IMF) and the Earth's magnetic field (Dungey, 1961; Gonzalez et al., 1994; Tsurutani et al., 1995; Zhang et al., 2007). The intensity and the orientation of the solar dipole is the source of the recurrent storms, while the size and the shape of the neutral sheet are the sources both of the quiet days and of the fluctuating activity (Simon and Legrand, 1989). However, there are no accurate expressions to clearly describe the relationship between the solar and geomagnetic activities.

The magnetic field is a crucial quantity to determine the state of the solar atmosphere and plays a key role in the formation and dynamics of solar activity. Sunspots $\left(R_{\mathrm{Z}}\right)$ represent one of the most obvious manifestations of local magnetic fields on the Sun. The solar activity affects the geomagnetic activity in various complex processes from the Sun to the Earth, involved in the solar - interplanetary - magnetospheric - ionospheric couplings (Tsurutani et al., 2006). The $a a$ geomagnetic index integrates all the effects on magnetosphere of several sources, such as solar flares, CMEs, and fast solar wind streams. The relationship between $a a$ and $R_{\mathrm{Z}}$ is not a simple linear or nonlinear function, rather it is an integral response function (10).

In this study, we presented a integral response model, $a a(t)=D \int_{t^{\prime}=-\infty}^{t} R_{\mathrm{Z}}\left(t^{\prime}\right) e^{-\left(t-t^{\prime}\right) / \tau} d t^{\prime}+a a_{0}$, to describe the relationship between the output (geomagnetic activity, $a a$ ) and the input (solar activity, $R_{\mathrm{Z}}$ ) of a (solar-terrestrial) system. In this model, the output $a a(t)$ depends not only on the present input $R_{\mathrm{Z}}(t)$, but also on past values. The output is a weighted sum of previous values of the input (Eqs. 2, 10 ), and the weights are given by the response function, $h\left(t-t^{\prime}\right) \propto e^{-\left(t-t^{\prime}\right) / \tau}$. The earlier the input, the less it contributes to the output. Parameter $D$ represents the "Dynamic response factor" of $a a$ to $R_{\mathrm{Z}}$ and reflects the linear correlation of $a a$ with $R_{\mathrm{z}}$. Parameter $\tau$ represents the "response time scale" of $a a$ to $R_{\mathrm{Z}}$ (it needs time for the energy transfer from solar to geomagnetic activity) and reflects the nonlinear correlation of $a a$ with $R_{\mathrm{z}}$. Parameter $a a_{0}$ represents the geomagnetic activity generated by earlier solar activities $\left(R_{\mathrm{z}}\right.$, solar flares, CMEs, shocks, and solar winds etc.). For each solar cycle the $a a_{0}$ value reflects and is well correlated with the geomagnetic minimum $\left(a a_{\min }\right)$.

For a linear relationship, the correlation coefficient between the $a a$ and $R_{\mathrm{Z}}$ series (3) is only $r_{0}=0.61$, implying that only about $r_{0}^{2}=37.2 \%$ of the variation in $a a$ can be explained by a linear dependence. However, when using our Model (10), the correlation between $a a$ and the reconstructed series $\left(a a_{\mathrm{f}}\right)$ for the overall data (Fig. 3) is much higher $\left(r_{\mathrm{f}}=\right.$ 0.85 ) than the above value, implying that about $r_{f}^{2}=72.3 \%$ of the variation in $a a$ can be explained by Model (10). If this model is applied to each solar cycle, the correlation will be even higher $\left(r_{\mathrm{f}}=0.95\right)$, implying that about $r_{f}^{2}=90.3 \%$ of the variation in $a a$ can be explained by Eq. (10). 

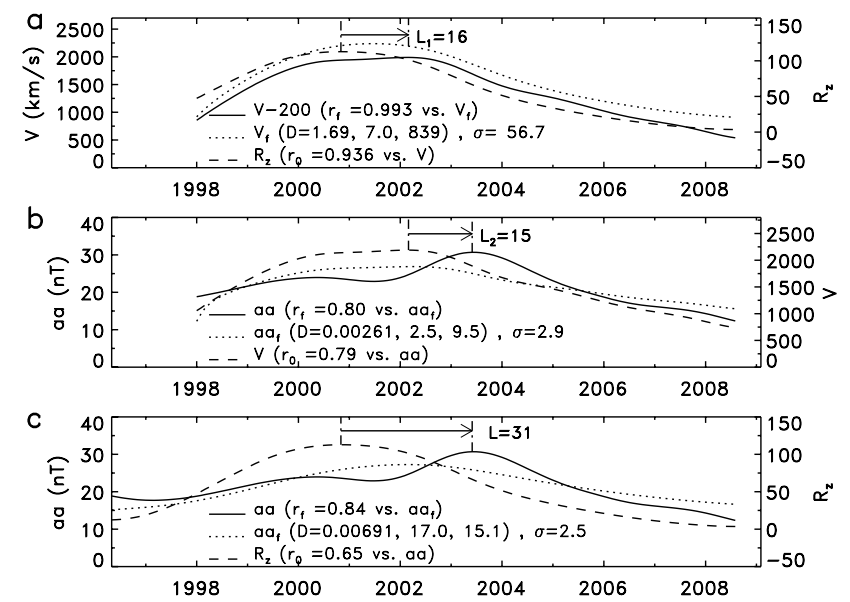

Fig. 8. (a) Monthly mean of integrated daily CME linear speed $V-$ 200 (solid, shifted downward by 200 for clarity) and $R_{\mathrm{Z}}$ (dashed) smoothed with a 24-month Gaussian filter. The dotted line shows the reconstructed series $\left(V_{\mathrm{f}}\right)$ from January 1998 to September 2008 by Model (10). The correlation coefficients of $V$ with $R_{\mathrm{Z}}$ and $V_{\mathrm{f}}$ are $r_{0}=0.936$ and $r_{\mathrm{f}}=0.993$, respectively. The lag time of $V$ to $R_{\mathrm{Z}}$ (at maximum) is $L_{1}=16$ (months). (b) Similar results for the relationship between $a a$ (solid) and $V$ (dashed). The correlation coefficients of $a a$ with $V$ and the reconstructed series $a a_{\mathrm{f}}$ are $r_{0}=$ 0.79 and $r_{\mathrm{f}}=0.80$, respectively. The lag time of $a a$ to $V$ is $L_{2}=$ 15 (months). (c) Similar results for the relationship between $a a$ (solid) and $R_{\mathrm{Z}}$ (dashed). The correlation coefficients of $a a$ with $R_{\mathrm{Z}}$ and the reconstructed series $a a_{\mathrm{f}}$ are $r_{0}=0.65$ and $r_{\mathrm{f}}=0.84$, respectively. The lag time of $a a$ to $R_{\mathrm{Z}}$ is $L=31$ (months). Other numbers indicate the fitted parameters $\left(D, \tau, y_{0}\right)$ and $\sigma$.

This model can naturally explain in part the time delay of $a a$ to $R_{\mathrm{Z}}$ and the following phenomena.

1. The significant increase in the $a a$ index over the twentieth century (Feynman and Crooker, 1978; Clilverd et al., 1998; Demetrescu and Dobrica, 2008; Lukianova et al., 2009), in either the cycle-averaged level or the baseline (Figs. 3, 4a, b and 6b).

2. The longer lag times of $a a$ to $R_{\mathrm{Z}}$ at solar maxima than at solar minima (Legrand and Simon, 1981; Wilson, 1990; Wang et al., 2000; Echer et al., 2004; Wang and Sheeley, 2009).

3. The stronger correlations between $a a$ and $R_{\mathrm{Z}}$ at rising phases than at declining phases (Du, 2011b).

4. The decreasing trend in the correlation between $a a$ and $R_{\mathrm{Z}}$ over time (Borello-Filisetti et al., 1992; Kishcha et al., 1999; Du, 2011b).

5. The increasing trend in the lag time of $a a$ to $R_{\mathrm{Z}}$ over time (Du, 2011b).

6. The turning point of the correlation of $a a$ with $R_{\mathrm{z}}$ around Cycle 19 (Du, 2011b).
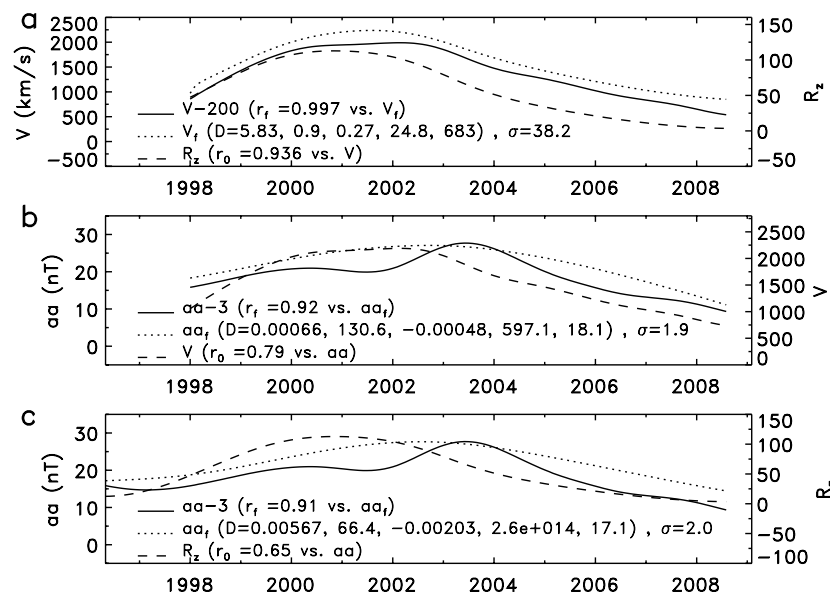

Fig. 9. Similar to Fig. 8 but using the double-decay model (12). (a) The correlation coefficient of $V$ (solid, shifted downward by 200 for clarity) with the reconstructed series $V_{\mathrm{f}}$ (dotted) from $R_{\mathrm{Z}}$ (dashed) by Eq. (12) is now $r_{\mathrm{f}}=0.997$. (b) The correlation coefficient of $a a$ (solid, shifted downward by 3 for clarity) with the reconstructed series $a a_{\mathrm{f}}$ (dotted) from $V$ (dashed) by Eq. (12) is now $r_{\mathrm{f}}=0.92$. (c) The correlation coefficient of $a a$ (solid, shifted downward by 3 for clarity) with the reconstructed series $a a_{\mathrm{f}}$ (dotted) from $R_{\mathrm{Z}}$ (dashed) by Eq. (12) is now $r_{\mathrm{f}}=0.91$.

7. The stronger correlations (and shorter lag times) at oddnumbered cycles than at the previous even-numbered cycles (Du, 2011b).

8. The stronger correlations (and shorter lag times) at even-numbered $H$-cycles than at the previous oddnumbered $H$-cycles (Du, 2011b).

The decreasing trend in the correlation between $a a$ and $R_{\mathrm{Z}}$ has ever been explained by the increasing occurrence of highspeed solar wind streams during the declining phase of solar cycle (Bame et al., 1976; Borello-Filisetti et al., 1992; Mussino et al., 1994; Tsurutani et al., 1995; Kishcha et al., 1999). However, during the declining phase of solar cycle, why does the occurrence of high-speed solar wind streams increase? In our Model (10), this trend is also due to the increasing trend in solar magnetic activity $\left(R_{\mathrm{Z}}\right)$ over the last century (Sect. 3.1).

In this study, we used the index of sunspot number $\left(R_{\mathrm{Z}}\right)$ to estimate the level of solar (magnetic) activity and its correlation with geomagnetic activity $(a a)$. We have not specified a special activity (e.g., solar wind) to describe the interaction process of solar-geomagnetic activities. In fact, magnetic fields play a determining role in the formation and dynamics of solar activity. The magnetic filed in solar corona (either close or open) is closely related to that on photosphere. Besides the sunspots, the magnetic fields may also produce other events of solar activities, such as solar flares, prominence eruptions, energetic protons, CMEs and solar winds (Legrand and Simon, 1989). Some of these events (e.g., 

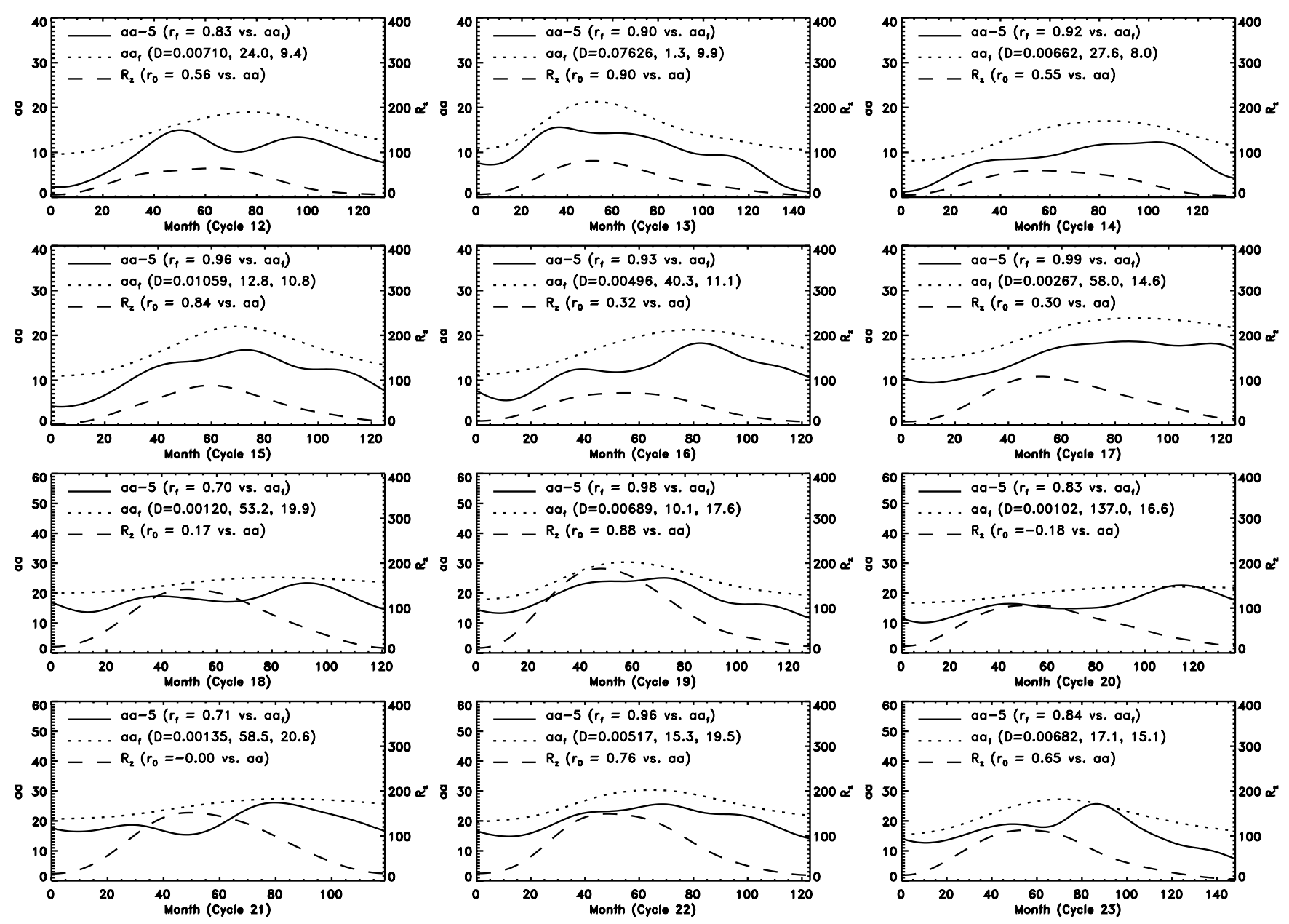

Fig. 10. Monthly mean $a a-5$ (solid, shifted downward by 5 for clarity) and $R_{\mathrm{Z}}$ (dashed) smoothed with a 24-month Gaussian filter, and the reconstructed $a a$ series $\left(a a_{\mathrm{f}}\right.$, dotted) by Model (10) for each cycle of $n=12$ to 23. In each plot are also shown the correlation coefficient of $a a$ with $R_{\mathrm{Z}}\left(r_{0}\right)$, that of $a a$ with $a a_{f}\left(r_{f}\right)$, and the three parameters of $D, \tau$ and $a a_{0}$.

solar flares) are produced soon or close in time to the formation of sunspots $\left(R_{\mathrm{Z}}\right)$. These events affect the geomagnetic fields $(a a)$ in a more linear manner. Some of other events (solar winds) are produced later than the formation of sunspots, whose effect on $a a$ is in a more nonlinear manner. These activities may be produced in different (nonlinear) processes more or less similar to Eq. (10), and play a role of mid-processes from the solar magnetic activity $\left(R_{\mathrm{Z}}\right)$ through coronal magnetic activity to geomagnetic activity $(a a)$. This may be the reasons why solar winds are later than $R_{\mathrm{z}}$ and occur often during declining phases, and why there are often more peaks in the $a a$ index. A similar process is also applicable to the relationships between $a a$ and these activities (solar flares, CMEs and solar winds). The effects of these processes are all integrated in Model (10).

As an example, we study the correlations of CME with solar activity $\left(R_{\mathrm{Z}}\right)$ and geomagnetic activity $(a a)$. Figure $8 \mathrm{a}$ shows the monthly mean of integrated daily CME linear speed $^{3} V$ (solid, shifted downward by 200 for clarity) and $R_{\mathrm{Z}}$ (dashed) smoothed with a 24-month Gaussian filter (Eq. 1). The dotted line shows the reconstructed series $\left(V_{\mathrm{f}}\right)$ from January 1998 to September 2008 based on Model (10). One can see that $V$ lags behind $R_{\mathrm{Z}}$ about $L_{1}=16$ (months) at the solar maximum and that the correlation coefficient of $V$ with the reconstructed series $V_{\mathrm{f}}\left(r_{\mathrm{f}}=0.993\right)$ is higher than that $\left(r_{0}=0.936\right)$ of $V$ with $R_{\mathrm{z}}$, meaning that about $98.6 \%$ (87.6\%) of the variation in $V$ can be explained by this model (linear dependence).

Figure $8 \mathrm{~b}$ shows the relationship between $a a$ (solid) and $V$ (dashed). One can also see that $a a$ lags behind $V$ about $L_{2}=15$ (months) and that the correlation coefficient of $a a$ with the reconstructed series $a a_{\mathrm{f}}\left(r_{\mathrm{f}}=0.80\right)$ by Model (10) is higher than that $\left(r_{0}=0.79\right)$ of $a a$ with $V$. Figure $8 \mathrm{c}$ shows the relationship between $a a$ (solid) and $R_{\mathrm{Z}}$ (dashed). The correlation coefficient of $a a$ with the reconstructed series $a a_{\mathrm{f}}$

\footnotetext{
${ }^{3}$ http://cdaw.gsfe.nasa.gov/CME_list/
} 
$\left(r_{\mathrm{f}}=0.84\right)$ by Model (10) is higher than that $\left(r_{0}=0.65\right)$ of $a a$ with $R_{\mathrm{z}}$. It should be pointed out that the lag time of $a a$ to $R_{\mathrm{Z}}$ ( $L=31$ months) is longer than the lag times of both $V$ to $R_{\mathrm{Z}}\left(L_{1}\right)$ and $a a$ to $V\left(L_{2}\right)$, and equal to $L_{1}+L_{2}$. This fact just reflects the energy transfer from solar activity $\left(R_{\mathrm{Z}}\right)$ through a mid-process (CME in this case) to geomagnetic activity $(a a)$. Similar conclusions may also hold for other solar activities (solar flares and solar winds etc) that have different lag times relative to $R_{\mathrm{z}}$ and that generate geomagnetic activities with different lag times relative to these activities.

As $R_{\mathrm{z}}$ and $a a$ are only rough estimates of solar and geomagnetic activities, respectively, the geomagnetic activity (aa) is only partially predictable by the $R_{\mathrm{z}}$ index. Besides, there are two peaks in either $a a$ or CME-related storms, one near the peak in $R_{\mathrm{z}}$ and another a few years later (Gonzalez and Tsurutani, 1987; Gonzalez et al., 1994, 2004; Tsurutani et al., 2006), while there is usually only one peak in Model (10) for a solar cycle. So this model can only describe the main profile of $a a$ and can not predict its fine structures. The fine structures are related to short-time variations in the sources of $a a$ (CMEs, solar winds and coronal holes etc.) and solar magnetic fields (in either solar surface or solar corona) as well. To improve the relationship between the output $y(t)$ and input $x(t)$, the following double-decay model can be used,

$$
\begin{aligned}
y(t) & =\int_{t^{\prime}=-\infty}^{t} x\left(t^{\prime}\right)\left[D_{1} e^{-\left(t-t^{\prime}\right) / \tau_{1}}+D_{2} e^{-\left(t-t^{\prime}\right) / \tau_{2}}\right] d t^{\prime}+y_{0} \\
& =\sum_{t^{\prime}=t_{0}}^{t} x\left(t^{\prime}\right)\left[D_{1} e^{-\left(t-t^{\prime}\right) / \tau_{1}}+D_{2} e^{-\left(t-t^{\prime}\right) / \tau_{2}}\right]+y_{0} .
\end{aligned}
$$

The results based on Model (12) using the data of Fig. 8 are shown in Fig. 9.

It is seen in Fig. 9a that the correlation coefficient of $V$ (solid, shifted downward by 200 for clarity) with the reconstructed series $V_{\mathrm{f}}$ (dotted) from $R_{\mathrm{Z}}$ (dashed) by Model (12) is now $r_{\mathrm{f}}=0.997$, slightly higher than that (0.993) of $V$ with the reconstructed series based on Model (10) in Fig. 8a. In Fig. 9b, the correlation coefficient of $a a$ (solid, shifted downward by 3 for clarity) with the reconstructed series $a a_{\mathrm{f}}$ (dotted) from $V$ (dashed) by Eq. (12) is now $r_{\mathrm{f}}=0.92$, higher than that $(0.80)$ of $a a$ with the reconstructed series by Model (10) in Fig. 8b. Figure 9c shows that the correlation coefficient $\left(r_{\mathrm{f}}=0.91\right)$ of $a a$ (solid, shifted downward by 3 for clarity) with the reconstructed series $a a_{\mathrm{f}}$ (dotted) from $R_{\mathrm{Z}}$ (dashed) by Eq. (12) is higher than that (0.84) of $a a$ with the reconstructed series by Model (10) in Fig. 8c.

The main profile of $a a$ can be well predicted from the $R_{\mathrm{Z}}$ series by Model (10). Even if using another more complex function, such as a geometric function of $a a(t)=$ $D \int_{t^{\prime}=-\infty}^{t} R_{\mathrm{Z}}^{\gamma}\left(t^{\prime}\right) e^{-\left(t-t^{\prime}\right) / \tau} d t^{\prime}+a a_{0}$ with an additional parameter $\gamma$, there will be no significant improvement in the correlation $\left(r_{\mathrm{f}}\right.$ increases from 0.85 to only 0.86$)$. Therefore, the relationship between $a a$ and $R_{\mathrm{z}}$ is mainly due to the integral response function. The relationship can be improved by the double-decay model (12). If considering two different lag times of $y$ to $x$ in Eq. (12), the result might be fur- ther improved. The remainder might be explained by other activities, such as nonlinear Alfvén waves, cosmic rays (Nagashima et al., 1991), and the interaction (Corotating Interaction Regions or CIRs) of fast with slow solar wind streams (Simon and Legrand, 1986; Richardson and Cane, 2002; Tsurutani et al., 2006), etc.

The main points of this paper may be summarized as follows,

1. An integral response model is proposed to describe the relationship between the geomagnetic index $(a a)$ and sunspot number $\left(R_{\mathrm{Z}}\right)$ : $a a(t)=$ $D \int_{t^{\prime}=-\infty}^{t} R_{\mathrm{Z}}\left(t^{\prime}\right) e^{-\left(t-t^{\prime}\right) / \tau} d t^{\prime}+a a_{0}$. Parameters $D$ and $\tau$ reflect the linear and nonlinear correlations of $a a$ with $R_{\mathrm{Z}}$, respectively. Parameter $a a_{0}$ represents the geomagnetic activity generated by earlier solar activities.

2. For all data from Cycles 12 through 23, the correlation coefficient of $a a$ with the reconstructed series based on this model $\left(r_{\mathrm{f}}=0.85\right)$ is much higher than the linear correlation coefficient $\left(r_{0}=0.61\right)$ of $a a$ with $R_{\mathrm{z}}$.

3. If this model is applied to each solar cycle, the correlation coefficient of $a a$ with the reconstructed series is higher $\left(r_{\mathrm{f}}=0.95\right)$. The $a a_{0}$ values reflects and is well correlated with the $a a$ minimum of solar cycle.

4. This model can naturally explain in part some phenomena related to the correlation of $a a$ with $R_{\mathrm{Z}}$, the lag time of $a a$ to $R_{\mathrm{z}}$, and their temporal variations.

Acknowledgements. The authors are grateful to the anonymous referees for suggestive and helpful comments. This work is supported by Chinese Academy of Sciences through grant YYYJ-1110, the National Natural Science Foundation of China (NSFC) through grants 10973020, 40890161 and 10921303, and National Basic Research Program of China through grants 2011CB811406.

Topical Editor P. Drobinski thanks two anonymous referees for their help in evaluating this paper.

\section{References}

Bachmann, K. T. and White, O. R.: Observations of hysteresis in solar cycle variations among seven solar activity indicators, Sol. Phys., 150, 347-357, 1994.

Bame, S. J., Asbridge, J. R., Feldman, W. C., and Gosling, J. T.: Solar cycle evolution of high-speed solar wind streams, Astrophys. J., 207, 977-980, 1976.

Borello-Filisetti, O., Mussino, V., Parisi, M., and Storini, M.: Longterm variations in the geomagnetic activity level. I - A connection with solar activity, Ann. Geophys., 10, 668-675, 1992, http://www.ann-geophys.net/10/668/1992/.

Cameron, R. and Schüssler, M.: Solar Cycle Prediction Using Precursors and Flux Transport Models, Astrophys. J., 659, 801-811, 2007.

Clilverd, E. W., Boriakoff, V., and Feynman, J.: Solar variability and climate change: Geomagnetic aa index and global surface temperature, Geophys. Res. Lett., 25, 1035-1038, 1998. 
Crooker, N. U., Feynman, J., and Gosling, J. T.: On the high correlation between long-term averages of solar wind speed and geomagnetic activity, J. Geophys. Res., 82, 1933-1937, 1977.

Demetrescu, C. and Dobrica, V.: Signature of Hale and Gleissberg solar cycles in the geomagnetic activity, J. Geophys. Res., 113, A02103, doi:10.1029/2007JA012570, 2008.

$\mathrm{Du}, \mathrm{Z}$. L.: The correlation between solar and geomagnetic activity - Part 1: Two-term decomposition of geomagnetic activity, Ann. Geophys., in review, 2011a.

Du, Z. L.: The correlation between solar and geomagnetic activity - Part 2: Long-term trends, Ann. Geophys., in review, 2011 b.

Du, Z. L. and Wang, H. N.: Is a higher correlation necessary for a more accurate prediction? Science China (Physics, Mechanics \& Astronomy), 54, 172-175, 2011.

Du, Z. L., Li, R., and Wang, H. N.: The Predictive Power of Ohl's Precursor Method, Astron. J., 138, 1998-2001, 2009.

Dungey, J. W.: Interplanetary magnetic field and the auroral zones, Phys. Rev. Lett., 6, 47-48, 1961.

Echer, E., Gonzalez, W. D., Gonzalez, A. L. C., Echer, E., Gonzalez, W. D., Gonzalez, A. L. C., Prestes, A., Vieira, L. E. A., dal Lago, A., Guarnieri, F. L., and Schuch, N. J.: Long-term correlation between solar and geomagnetic activity, J. Atmos. Sol. Terr. Phys., 66, 1019-1025, 2004.

Feynman, J.: Implications of solar cycles 19 and 20 geomagnetic activity for magnetospheric processes, Geophys. Res. Lett., 7, 971-973, 1980.

Feynman, J.: Geomagnetic and solar wind cycles, 1900-1975, J. Geophys. Res., 87, 6153-6162, 1982.

Feynman, J. and Crooker, N. U.: The solar wind at the turn of the century, Nature, 275, 626-627, 1978.

Garrett, H. B., Dessler, A. J., and Hill, T. W.: Influence of solar wind variability on geomagnetic activity, J. Geophys. Res., 79, 4603-4610, 1974.

Gnevyshev, M. N. and Ohl, A. I.: On the 22-year solar activity cycle, Astron. Z., 25, 18-20, 1948.

Gonzalez, W. D. and Tsurutani, B. T.: Criteria of interplanetary parameters causing intense magnetic storms (Dst of less than -100 nT), Planet. Space Sci., 35, 1101-1109, 1987.

Gonzalez, W. D., Gonzalez, A. L. C., Tsurutani, B. T., Smith, E. J., and Tang, F.: Solar wind-magnetosphere coupling during intense magnetic storms (1978-1979), J. Geophys. Res., 94, 8835-8851, 1989.

Gonzalez, W. D., Joselyn, J. A., Kamide, Y., Kroehl, H. W., Rostoker, G., Tsurutani, B. T., and Vasyliunas, V. M.: What is a geomagnetic storm?, J. Geophys. Res., 99, 5771-5792, 1994.

Gonzalez, W. D., Dal Lago, A., Clua de Gonzalez, A. L., Vieira, L. E. A., and Tsurutani, B. T.: Prediction of peak-Dst from halo CME-magnetic cloud-speed observations, J. Atmos. Sol. Terr. Phys., 66, 161-165, 2004.

Gosling, J. T. and Pizzo, V. J.: Formation and evolution of corotating interaction regions and their three dimensional strcture, Space Sci. Rev., 89, 21-52, 1999.

Hathaway, D. H. and Wilson, R. M.: Geomagnetic activity indicates large amplitude for sunspot cycle 24, Geophys. Res. Lett., 33, L18101, doi:10.1029/2006GL027053, 2006.

Hathaway, D. H., Wilson, R. M., and Reichmann, E. J.: Group sunspot numbers: sunspot cycle characteristics, Solar Phys., 211, 357-370, 2002.

Johnson, J. R. and Wing, S.: A solar cycle dependence of nonlinear- ity in magnetospheric activity, J. Geophys. Res., 110, A04211, doi:10.1029/2004JA010638, 2005.

Kishcha, P. V., Dmitrieva, I. V., and Obridko, V. N.: Long-term variations of the solar - geomagnetic correlation, total solar irradiance, and northern hemispheric temperature (1868-1997), J. Atmos. Sol. Terr. Phys., 61, 799-808, 1999.

Legrand, J. P. and Simon, P. A.: Ten cycles of solar and geomagnetic activity, Solar Phys., 70, 173-195, 1981.

Legrand, J. P. and Simon, P. A.: Solar cycle and geomagnetic activity: A review for geophysicists. I - The contributions to geomagnetic activity of shock waves and of the solar wind, Ann. Geophys., 7, 565-578, 1989, http://www.ann-geophys.net/7/565/1989/.

Lockwood, M., Stamper, R., and Wild, M. N.: A doubling of the Sun's coronal magnetic field dring the past 100 years, Nature, 399, 437-439, 1999.

Lukianova, R., Alekseev, G., and Mursula, K.: Effects of station relocation in the aa index, J. Geophys. Res., 114, A02105, doi:10.1029/2008JA013824, 2009.

Mayaud, P. N.: The aa indices: A 100-year series characterizing the magnetic activity, J. Geophys. Res., 77, 6870-6874, 1972.

Moradi, H., Baldner, C., Birch, A. C., Braun, D. C., Cameron, R. H., Duvall Jr., T. L., Gizon, L., Haber, D., Hanasoge, S. M., Hindman, B. W., Jackiewicz, J., Khomenko, E., Komm, R., Rajaguru, P., Rempel, M., Roth, M., Schlichenmaier, R., Schunker, H., Spruit, H. C., Strassmeier, K. G., Thompson, M. J., and Zharkov, S.: Modeling the Subsurface Structure of Sunspots, Solar Phys., 267, 1-62, 2010.

Mursula, K., Martini, D., and Karinen, A.: Did open solar magnetic field increase during the last 100 years? A reanalysis of geomagnetic activity, Solar Phys., 224, 85-94, 2004.

Mussino, V., Borello Filisetti, O., Storini, M., and Nevanlinna, H.: Long-term variations in the geomagnetic activity level Part II: Ascending phases of sunspot cycles, Ann. Geophys., 12, 10651070, doi:10.1007/s00585-994-1065-5, 1994.

Nagashima, K., Fujimoto, K., and Tatsuoka, R.: Nature of solarcycle and heliomagnetic-polarity dependence of cosmic rays, inferred from their correlation with heliomagnetic spherical surface harmonics in the period 1976-1985, Planet. Space Sci., 39, 1617-1635, 1991.

Nevanlinna, H. and Kataja, E.: An extension of the geomagnetic activity index series aa for two solar cycles (1844-1868), Geophys. Res. Lett., 20, 2703-2706, 1993.

Parker, E. N.: Interplanetary Dynamical Processes, Interscience Publishers, New York, 1963.

Penn, M. J. and Livingston, W.: Temporal Changes in Sunspot Umbral Magnetic Fields and Temperatures, Astrophys. J., 649, L45L48, 2006.

Prestes, A., Rigozo, N. R., Echer, E., and Vieira, L. E. A.: Spectral analysis of sunspot number and geomagnetic indices (18682001), J. Atmos. Sol. Terr. Phys., 68, 182-190, 2006.

Richardson, I. G. and Cane, H. V.: Sources of geomagnetic activity during nearly three solar cycles (1972-2000), J. Geophys. Res., 107(A8), SSH 8-1, 1187, doi:10.1029/2001JA000504, 2002.

Russell, C. T. and McPherron, R. L.: Semiannual variation of geomagnetic activity, J. Geophys. Res., 78, 92-108, 1973.

Russell, C. T. and Mulligan, T.: The 22-year variation of geomagnetic activity: Implications for the polar magnetic field of the Sun, Geophys. Res. Lett., 22, 3287-3288, 1995. 
Sakurai, T.: Calculation of force-free magnetic field with nonconstant $\alpha$, Solar Phys., 69, 343-359, 1981.

Sargent, H. H.: Recurrent geomagnetic activity - Evidence for long-lived stability in solar wind structure, J. Geophys. Res., 90, 1425-1428, 1985.

Schatten, K. H. and Pesnell, W. D.: An early solar dynamo prediction: Cycle 23 is approximately cycle 22, Geophys. Res. Lett., 20, 2275-2278, 1993.

Schatten, K. H., Scherrer, P. H., Svalgaard, L., and Wilcox, J. M.: Using dynamo theory to predict the sunspot number during solar cycle 21, Geophys. Res. Lett., 5, 411-414, 1978.

Schwenn, R.: Solar wind sources and their variations over the solar cycle, Space Sci. Rev., 124, 51-76, 2006.

Simon, P. A. and Legrand, J. P.: Some solar cycle phenomena related to the geomagnetic activity from 1868 to 1980, Astron. Astrophys., 155, 227-236, 1986.

Simon, P. A. and Legrand, J. P.: Solar cycle and geomagnetic activity: A review for geophysicists. II - The solar sources of geomagnetic activity and their links with sunspot cycle activity, Ann. Geophys., 7, 579-593, 1989.

Snyder, C. W., Neugebauer, M., and Rao, U. R.: The Solar Wind Velocity and Its Correlation with Cosmic-Ray Variations and with Solar and Geomagnetic Activity, J. Geophys. Res., 68, 63616370, 1963.

Stamper, R., Lockwood, M., Wild, M. N., and Clark, T. D. G.: Solar causes of the long-term increase in geomagnetic activity, J. Geophys. Res., 104, 28325-28342, 1999.

Svalgaard, L.: Geomagnetic activity: Dependence on solar wind parameters, in: Coronal Holes and High Speed Wind Streams, edited by: Zirker, J. B., Colorado Ass. U. Press, Boulder, p. 371, 1977.

Temmer, M., Veronig, A., and Hanslmeier, A.: Does solar flare activity lag behind sunspot activity? Solar Phys., 215, 111-126, 2003.

Tsurutani, B. T., Gonzalez, W. D., Tang, F., Akasofu, S. I., and Smith, E. J.: Origin of interplanetary southward magnetic fields responsible for major magnetic storms near solar maximum (1978-1979), J. Geophys. Res., 93, 8519-8531, 1988.
Tsurutani, B. T., Gonzalez, E. D., Gonzalez, A. L. C., Tang, F., Arballo, J. K., and Okada, M.: Interplanetary origin of geomagnetic activiy in the declining phase of the solar cycle, J. Geophys. Res., 100, 21717-21733, 1995.

Tsurutani, B. T., Gonzalez, W. D., Gonzalez, A. L. C., Guarnieri, F. L., Gopalswamy, N., Grande, M., Kamide, Y., Kasahara, Y., Lu, G., Mann, I., McPherron, R., Soraas, F., and Vasyliunas, V.: Corotating solar wind streams and recurrent geomagnetic activity: A review, J. Geophys. Res., 111, A07S01, doi:10.1029/2005JA011273, 2006.

Tu, C. Y. and Marsch, E.: MHD structures, waves and turbulence in the solar wind: observations and theories, Space Sci. Rev., 73, 1-210, 1995.

Venkatesan, D., Ananth, A. G., Graumann, H., and Pillai, S.: Relationship between solar and geomagentic activity, J. Geophys. Res., 96, 9811-9813, 1991.

Wang, Y. M. and Sheeley, N. R.: Understanding the Geomagnetic Precursor of the Solar Cycle, Astrophys. J., 694, L11-L15, 2009.

Wang, Y. M., Lean, J., and Sheeley, N. R.: The long-term variation of the Sun's open magnetic flux, Geophys. Res. Lett., 27, 505508, 2000.

Wheatland, M. S. and Litvinenko, Y. E.: Energy balance in the flaring solar coronaA, Astrophys. J., 557, 332-336, 2001.

Wilson, R. M.: Bimodality and the Hale cycle, Solar Phys., 117, 269-278, 1988.

Wilson, R. M.: On the level of skill in predicting maximum sunspot number - A comparative study of single variate and bivariate precursor techniques, Solar Phys., 125, 143-155, 1990.

Yan, Y. and Li, Z.: Direct Boundary Integral Formulation for Solar Non-constant- $\alpha$ Force-free Magnetic Fields, Astrophys. J., 638, 1162-1168, 2006.

Zhang, J., Richardson, I. G., Webb, D. F., Gopalswamy, N., Huttunen, E., Kasper, J. C., Nitta, N. V., Poomvises, W., Thompson, B. J., Wu, C.-C., Yashiro, S., and Zhukov, A. N.: Solar and interplanetary sources of major geomagnetic storms (Dst $<=$ $-100 \mathrm{nT}$ ) during 1996-2005, J. Geophys. Res., 112, A10102, doi:10.1029/2007JA012321, 2007. 